\title{
Emission gain narrowing from single crystals of a thiophene/phenylene co-oligomer
}

\author{
Michifumi Nagawa and Ryota Hibino \\ Department of Functional Polymer Science, Faculty of Textile Science and Technology, Shinshu University, \\ 3-15-1 Tokita, Ueda 386-8567, Japan \\ Shu Hotta \\ Joint Research Center for Harmonized Molecular Materials (JRCHMM)-Japan Chemical Innovation \\ Institute (JCII), clo National Institute of Advanced Industrial Science and Technology (AIST), Tsukuba \\ Central 5, 1-1-1 Higashi, Tsukuba, Ibaraki 305-8565, Japan \\ Hisao Yanagi \\ Faculty of Engineering, Kobe University, Rokkodai, Nada, Kobe 657-8501, Japan \\ Musubu Ichikawa, Toshiki Koyama, and Yoshio Taniguchi ${ }^{\text {a) }}$ \\ Department of Functional Polymer Science, Faculty of Textile Science and Technology, Shinshu University, \\ 3-15-1 Tokita, Ueda 386-8567, Japan
}

(Received 30 August 2001; accepted for publication 27 November 2001)

Emission gain narrowing has been observed for single crystals of a thiophene/phenylene co-oligomer. The hexagon flake crystals were placed on a quartz substrate with the crystals' face in close contact with the substrate plane. These crystals were irradiated with a $\mathrm{N}_{2}$ laser with a 337.1 $\mathrm{nm}$ wavelength at a repetition rate $10 \mathrm{~Hz}$ that tuned its intensity to $100-1150 \mu \mathrm{J} / \mathrm{cm}^{2}$. The emission gain narrowing takes place at $21490(465.4 \mathrm{~nm})$ and $20220 \mathrm{~cm}^{-1}(494.5 \mathrm{~nm})$ with increased intensities, with their half width at half maxima reaching $\sim 50 \mathrm{~cm}^{-1}$. On the basis of the nonlinear relationship between the emission peak intensities and the laser light intensity, the gain narrowing has been attributed to the amplified spontaneous emission. (C) 2002 American Institute of Physics. [DOI: $10.1063 / 1.1435797]$

Conjugated semiconducting polymers and oligomers are currently attracting great attention as potentially useful materials for optoelectronic devices such as thin-film transistors and light-emitting diodes. ${ }^{1}$ Solid-state lasing and amplified spontaneous emission (ASE) have been observed in these materials. ${ }^{2}$ Since excitation through photopumping is a facile way to attain lasing and ASE, this method has long been used. $^{3-5}$ The earliest examples can be found for anthracene molecules embedded in host matrices of, e.g., fluorene. ${ }^{6}$ Later this approach was more widely applied to dye-doped systems and polymer thin films. ${ }^{7}$

Very recently solid-state injection lasing was achieved using a tetracene single crystal, vouching for the high practicality of organic semiconductors. ${ }^{8}$ In view of current injection, crystals of high quality are more advantageous than any other systems that involve defects which may well act as carrier traps. Reported observation of lasing or ASE of the crystals, however, remains scarce even in the case of photopumping. This is partly because the photoluminescent efficiency of chromophores is decreased by higher concentrations, ${ }^{9}$ even though their efficiency is high at dilution. Yet a typical examples for the ASE haves been verified by Fichou et al. ${ }^{10}$ and by Horowitz et al. ${ }^{11}$ using single crystals of octithiophene and sexithiophene, respectively.

Yanagi and Morikawa ${ }^{12}$ showed that self-waveguided emission takes place along the crystals' long axis of needleshaped crystals of $p$-sexiphenyl that are epitaxially grown on

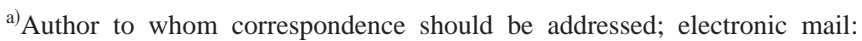
tany@giptc.shinshu-u.ac.jp
}

top of a single crystal substrate of potassium chloride. The molecular axes of $p$-sexiphenyl align perpendicular to the crystals' needle axis so that the propagation of the polarized emission can be enhanced with the transverse electric mode along that needle. The uniaxially aligned transition dipoles in the $p$-sexiphenyl crystals are responsible for the selfwaveguided emission. Recent progress demonstrated that this is analogous to the case of a newly emerging class of semiconducting molecular crystals, thiophene/phenylene co-oligomers. ${ }^{13}$ These materials were developed by Hotta and co-workers ${ }^{14}$ and are characterized by a variety of extensions of $\pi$ conjugation along the backbone. The conjugation extension can be tuned by changing the total number of thiophenes and phenylenes and their arrangement in the molecules. Since self-waveguided propagation of the emission is a prerequisite for lasing or ASE, the thiophene/phenylene co-oligomers are expected to be good candidates for organic laser materials. In this letter we report initial results of the ASE, which has been observed for a co-oligomer BP1T [see its structural formula in Fig. 1(a)].

The experimental setup for the optical measurements is schematically represented in Fig. 2. In Fig. 2 the crystals were irradiated with a $\mathrm{N}_{2}$ laser with a $337.1 \mathrm{~nm}$ wavelength at a repetition rate $10 \mathrm{~Hz}$. Laser light of $1 \mathrm{~mm} \times 5 \mathrm{~mm}$ was perpendicularly incident on a quartz substrate. The hexagon crystals of typically $\sim 100 \mu \mathrm{m}$ and thickness of $\sim 5 \mu \mathrm{m}$ [see Fig. 1(b)] were dispersed in water and this dispersion was drop cast onto the substrate and subsequently dried. These flake crystals showed good adhesion to the substrate with the crystals' face in close contact with the substrate plane. Thus 
(a)

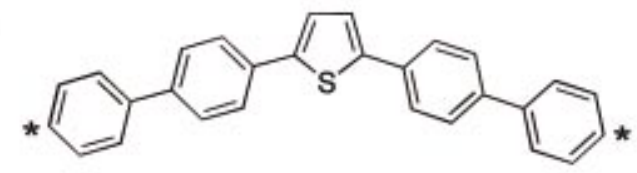

(b)

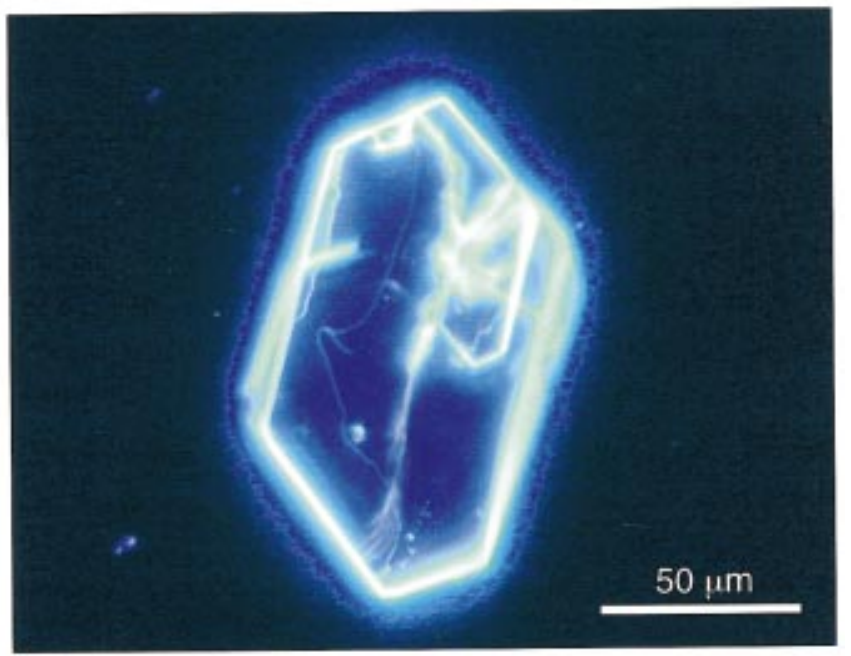

FIG. 1. (Color) (a) Structural formula of BP1T. The four carbons located on the terminal phenyls are indicated by asterisks. (b) Micrograph of a BP1T crystal.

several crystallites were irradiated at once with the rectangular laser beam. The intensity of the excitation beam was regulated from 100 to $1150 \mu \mathrm{J} / \mathrm{cm}^{2}$ using a ND filter. The light emitted from the crystal edges was collected along the direction parallel to the substrate plane. That emitted light was detected through a UV cut filter with an Oriel MS-127I spectrometer connected to an Andor ICCD V charge-coupled imaging device. Standard $\mathrm{x}$-ray diffraction (XRD) measurements $(\theta-2 \theta)$ were carried out using $\mathrm{Cu} K \alpha$ radiation.

The XRD measurements of the "on-substrate" crystals

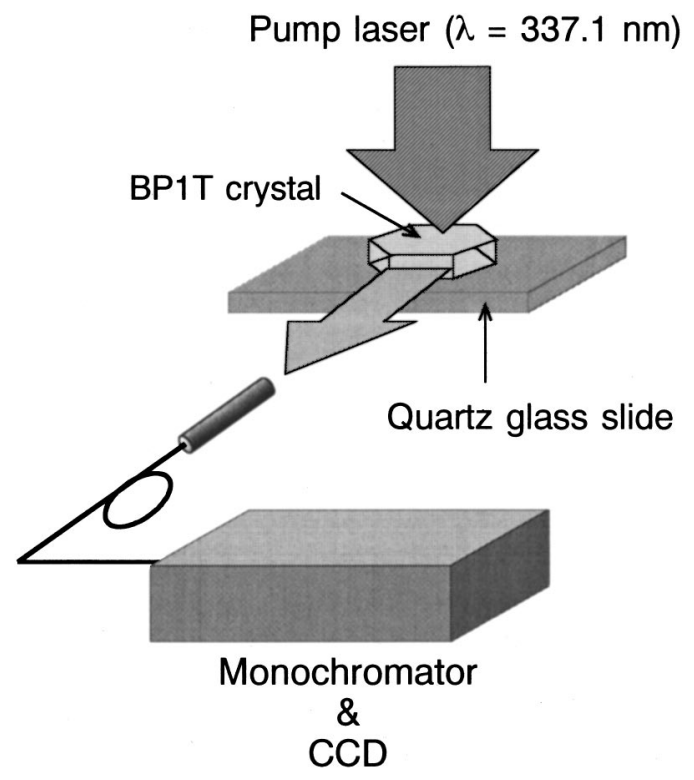

FIG. 2. Schematic of the experimental setup for optical measurements. D. 2. Schenatic of the experinental setup for optical measurene

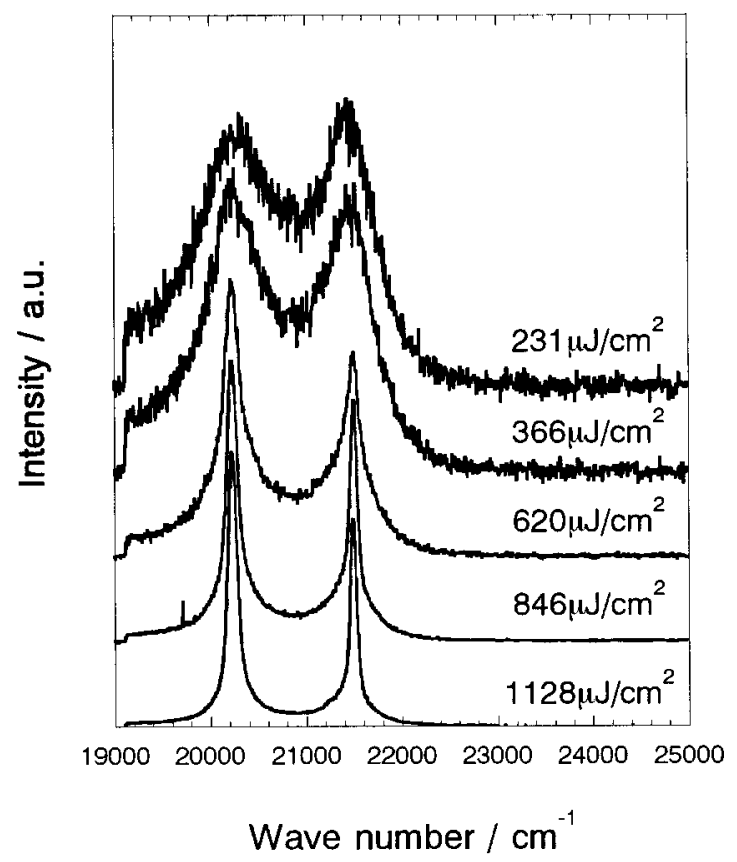

FIG. 3. Emission spectra of BP1T crystals as a function of the intensity of the incident laser light.

indicate that the diffractions comprise primary diffraction spacing of $\sim 2.2 \mathrm{~nm}$ and its higher order reflections. ${ }^{15}$ This means that the crystals consist of a molecular layered structure with the "molecular long axis" exhibiting vertical alignment against the substrate plane. ${ }^{15}$ (vide infra).

Under weaker incident intensities the emission spectra exhibit a broad feature with relatively fine modes superimposed on it. These fine modes can be assigned to the vibronic structures that are frequently observed in solid-phase molecules with well-defined molecular symmetry. ${ }^{16}$ Of these vibronic modes, only a few specific modes are strongly narrowed by an increase in incident intensities. Figure 3 shows emission spectra from the BP1T crystals as a function of the intensity of the incident laser light. In Fig. 3 two lines are clearly narrowe at $21490(465.4 \mathrm{~nm})$ and $20220 \mathrm{~cm}^{-1}(494.5$ $\mathrm{nm})$. At intensity of $366 \mu \mathrm{J} / \mathrm{cm}^{2}$ the half widths at half maximum (HWHMs) of the former and latter lines were 276 and $381 \mathrm{~cm}^{-1}$, respectively. With an increase in intensity the HWHMs rapidly became narrowe so that they were 51 and $46 \mathrm{~cm}^{-1}$ for the 21490 and $20220 \mathrm{~cm}^{-1}$ lines, respectively. At the same time, the peak intensities rapidly increased as well. Figure 4 presents the HWHMs and photoluminescence (PL) peak intensities as a function of the incident laser light intensity. When the incident laser intensity is increased, the PL peak intensities grow nonlinearly, accompanied by the narrowing shown in Fig. 4. Therefore, we conclude that the narrowing is caused by gain narrowing, namely, ASE. ${ }^{9-11} \mathrm{We}$ present the extent of the gain narrowing in terms of the HWHM, because the positions of the gain-narrowed lines are not fully resolved at weaker excitation intensities (see Fig. $3)$. The HWHM approaches half of the full width at half maximum (FWHM) with stronger excitation intensities. The linewidths are usually measured with the FWHM. Compare the above-mentioned HWHMs $\left(\sim 50 \mathrm{~cm}^{-1}\right)$ with the FWHM $\left(\sim 170 \mathrm{~cm}^{-1}\right)$ determined by Fichou et al. for octithiophene crystals. ${ }^{10}$ Note here that the linewidth of gain-
to AIP license or copyright, see http://ojps.aip.org/aplo/aplcr.jsp 


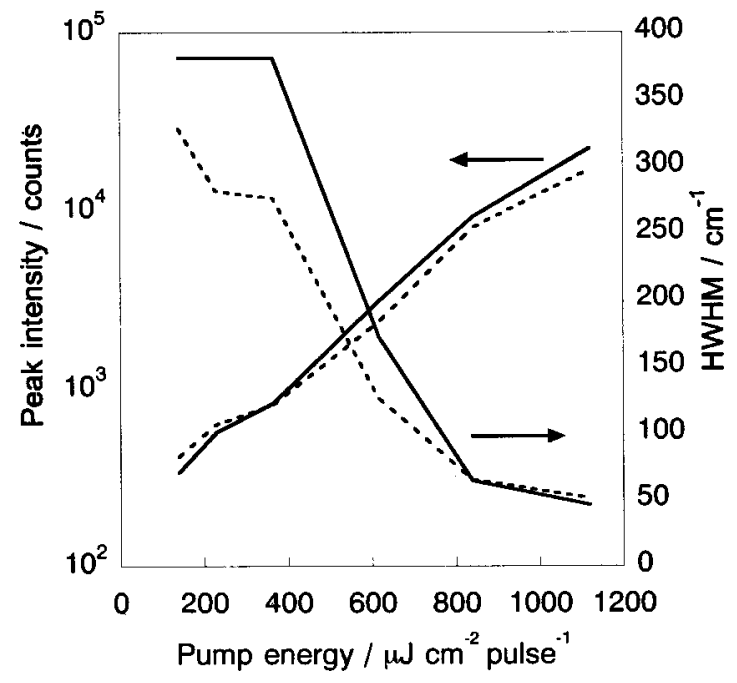

FIG. 4. HWHMs and PL peak intensities as a function of the incident laser light intensity. Solid and dashed lines represent 20220 and $21490 \mathrm{~cm}^{-1}$ lines, respectively.

narrowed emissions by ASE is governed by many factors, for example, the linewidth of spontaneous emission. ${ }^{17}$

The relevance of the crystal and molecular structures of BP1T to the ASE observed is worth briefly mentioning. In the crystals the deviation from $C_{2 v}$ symmetry is relatively small, ${ }^{18}$ so the transition dipoles are likely to be parallel to the line connecting the four carbons located on the terminal phenyls [see Fig. 1(a)]. ${ }^{19}$ This line represents the molecular long axis. The angle between said line and the normal of the $a b$ plane of the crystals (that parallels to the crystal faces) is roughly $1{ }^{\circ} \cdot{ }^{18}$ Since the $a b$ plane parallels the crystals' faces, the transition dipoles are nearly upright against them (and the substrate plane also). In this vertical dipole configuration, the light emissivity from the molecules is maximum in the direction parallel to the crystals' faces and amplification of self-waveguided light propagating in this direction can readily be attained.

With Regard to the excitation experiments the two lines were narrowed to a similar extent at once in some cases, as can be seen in Fig. 3. In other cases, on the other hand, either the longer wavelength line or the shorter wavelength one was preferentially gain narrowed according to the individual measurements. In this context, Fichou et al. ${ }^{10}$ observed related narrowing trends for $\alpha$-octithiophene crystals and pointed out that those trends are associated with the sample size and irradiated location of the crystals. In other words, they carried out the experiments such that a crystal $1-3 \mathrm{~mm}$ long was irradiated with a focused beam of diameter of $\sim 0.3 \mathrm{~mm}$ and its position was scanned along the crystals' length. What was observed was that the $700 \mathrm{~nm}$ line was gain narrowed over most of the crystals' surface while the $640 \mathrm{~nm}$ line was almost inactive with gain narrowing except near the crystals' edge or on a second single crystal stacked on top of the main one. Note that here the experimental conditions in our studies are totally different from those of Fichou et al. ${ }^{10}$ As mentioned earlier, we used conditions in which several small crystallites were irradiated at once. Since the individual crystallites contained further smaller ones on their surface [Fig. 1(b)], the main crystals and secondary smaller ones were irradiated all together. Consequently, it follows that we have observed collective PL from crystals of different size. The question of how the relevant lines are gain-narrowed under each specific condition, however, needs thorough investigating by accumulating more data using more materials.

In conclusion, we have investigated the emission gain narrowing of BP1T single crystals. The gain-narrowed emissions take place as a consequence of waveguided propagation of the light emitted and are associated with ASE. The disposition of the transition dipoles optimizes this selfwaveguided ASE that occurrs as edge emission. This is expected to lead to lasing under suitable conditions.

The authors thank Dr. Y. Yoshida, National Institute of Advanced Industrial Science and Technology (AIST), for making the XRD measurements. This work was supported by NEDO for the Harmonized Molecular Materials theme funded through the project on Technology for Novel HighFunctional Materials (AIST). This work was also supported by NEDO for the Organic Materials Technology for a SolidState Injection Laser theme.

${ }^{1}$ D. Braun and A. J. Heeger, Appl. Phys. Lett. 66, 2540 (1995).

${ }^{2}$ R. Gupta, M. Stevenson, A. Dogariu, M. D. McGehee, J. Y. Park, V. Srdanov, A. J. Heeger, and H. Wang, Appl. Phys. Lett. 73, 3492 (1998).

${ }^{3}$ M. Berggren, A. Dodabalapur, and R. E. Slusher, Appl. Phys. Lett. 71, 2230 (1997)

${ }^{4}$ V. G. Kozlov, V. Bulovic, P. E. Burrows, and S. R. Forrest, Nature (London) 389, 362 (1997).

${ }^{5}$ M. Nagawa, M. Ichikawa, T. Koyama, H. Shirai, Y. Taniguchi, A. Hongo, S. Tsuji, and Y. Nakano, Appl. Phys. Lett. 77, 2641 (2000).

${ }^{6}$ N. Karl, J. Lumin. 12/13, 851 (1976).

${ }^{7}$ Y. C. Kim, T.-W. Lee, O. O. Park, C. Y. Kim, and H. N. Cho, Adv. Mater. 13, 646 (2001).

${ }^{8}$ J. H. Schön, Ch. Kloc, A. Dodabalapur, and B. Batlogg, Science 289, 599 (2000).

${ }^{9}$ M. D. McGehee and A. J. Heeger, Adv. Mater. 12, 1655 (2000).

${ }^{10}$ D. Fichou, S. Delysse, and J.-M. Nunzi, Adv. Mater. 9, 1178 (1997).

${ }^{11}$ G. Horowitz, P. Valat, F. Garnir, F. Kouki, and V. Wintgens, Opt. Mater. 9, 46 (1998).

${ }^{12}$ H. Yanagi and T. Morikawa, Appl. Phys. Lett. 75, 187 (1999).

${ }^{13}$ H. Yanagi, T. Morikawa, S. Hotta, and K. Yase, Adv. Mater. 13, 313 (2001)

${ }^{14}$ S. A. Lee, Y. Yoshida, M. Fukuyama, and S. Hotta, Synth. Met. 106, 39 (1999).

${ }^{15}$ S. Hotta, Y. Ichino, Y. Yoshida, and M. Yoshida, J. Phys. Chem. B 104, 10316 (2000).

${ }^{16}$ S. Hotta and K. Waragai, Adv. Mater. 5, 896 (1993).

${ }^{17}$ L. W. Casperson and A. Yariv, IEEE J. Quantum Electron. 8, 80 (1972).

${ }^{18} \mathrm{~S}$. Hotta (unpublished data).

${ }^{19}$ F. A. Cotton, Chemical Applications of Group Theory, 2nd ed. (Wiley, New York, 1971), Chap. 7; Appendix III A. 